\title{
Effects of resveratrol, an important component of red wine, on intestinal cancer development
}

\author{
Xiaoying Zhang' \\ Jan Anderson' \\ Radhey S Kaushik ${ }^{2,3}$ \\ Chandradhar Dwivedi' \\ 'Department of Pharmaceutical \\ Sciences; ${ }^{2}$ Department of Veterinary \\ Sciences; ${ }^{3}$ Department of Biology/ \\ Microbiology, South Dakota State \\ University, Brookings, SD, USA
}

\begin{abstract}
Resveratrol, a natural product derived from grapes and an important component of red wine, has been shown to inhibit cyclooxygenase and prevent various cancers. The purpose of this study is to investigate the effects of dietary grape extract, a source of resveratrol on intestinal cancer development in rats and to determine effects of resveratrol on cell growth in human colonic adenocarcinoma (Caco-2) cells, thus elucidating possible mechanisms of action of resveratrol. Results showed that dietary grape extract ( $5 \%$, about $7 \mu \mathrm{g}$ resveratrol consumed daily) significantly decreased the incidence and multiplicity of tumors in small intestine in rats and resveratrol significantly inhibited cell viability and cell proliferation in Caco-2 cells.
\end{abstract}

Keywords: resveratrol, grapes, colonic adenocarcinoma, Caco-2 cells

\section{Introduction}

Resveratrol (trans-3,5,4'-trihydroxystilbene) (Figure 1), a polyphenolic phytoalexin, is an important component of red wine and other grape products. This compound is abundant in grape wines, but is relatively low in fruits and vegetables. ${ }^{1}$ Resveratrol is produced preferentially by fungal infection and the resveratrol content of wine is related to the length of time that the grape skins are present during the fermentation process. The grape skins are removed earlier during white wine production than that of red wine; thus, the concentration of resveratrol in red wine is much higher than in white wine. ${ }^{2}$

In recent years, studies suggest that the consumption of wine, particularly of red wine, reduces the incidence and morbidity of coronary heart disease. ${ }^{3,4}$ Resveratrol derived from grapes was found to act as an antioxidant and antimutagen, induces phase II drug metabolizing enzymes, mediates anti-inflammatory effects, and induces promyelocytic leukemia cell differentiation. ${ }^{5}$ Resveratrol exhibits anticancer properties by mediating apoptosis, ${ }^{6-8}$ arresting cell cycle progression, ${ }^{9}$ antiproliferation ${ }^{8}$ and inhibiting ribonucleotide reductase, ornithine decarboxylase (ODC), and cyclooxygenase (COX-2) through modulation of prostaglandin production. ${ }^{10-13}$ Studies have shown that COX-2 is overexpressed in almost $90 \%$ of human colorectal cancers ${ }^{14}$ and nonsteroidal anti-inflammatory drugs (NSAIDs) have been widely studied as possible chemopreventive agents in colon cancer development. ${ }^{15}$ For example, long-term use of aspirin and other NSAIDs has shown a reduction of the risk of colon cancer and lower mortality in clinical case control studies. ${ }^{15}$ Accordingly, inhibition of COX-2 could be an effective approach for the chemoprevention of colorectal cancers. ${ }^{16}$

Colorectal cancers are the third most prevalent cancers in the United States and account for $10 \%$ of cancer deaths in both men and women. An estimated 108,070 new cases of colon cancer and 40,740 new cases of rectal cancer are expected to be diagnosed, and 49,960 colorectal cancer-related deaths are expected to occur in 2008. ${ }^{17}$ Worldwide colorectal cancer affects over one million people every year and is responsible for more than a half million cancer-related annual deaths. Generally, colon cancer occurs in individuals older than 50 years and develops as a 


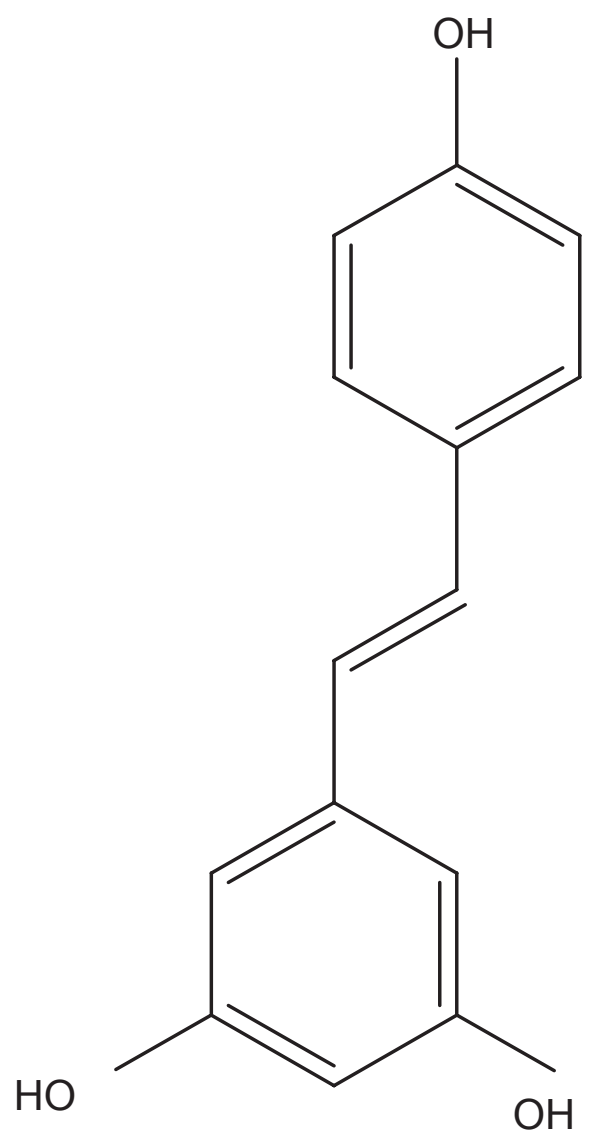

Figure I Stucture of trans-resveratrol.

consequence of environmental carcinogens and/or genetic factors. Lifestyle factors including diet, overweight, low physical activity, and smoking may account for $70 \%$ of

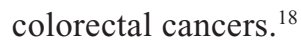

However, effects of resveratrol on colon cancer development in animal models have not been well studied. Thus, the objective of this study is to investigate the effects of dietary grape extract $(5 \%)$, a dietary source of resveratrol on azoxymethane-induced intestinal cancer development in male Fischer rats. Cancer is a disease caused by uncontrolled cell growth. ${ }^{19}$ Accordingly, the effects of resveratrol on cell proliferation in human colonic adenocarcinoma (Caco-2) cells were also investigated to elucidate the possible mechanism of action of resveratrol.

\section{Materials and method}

\section{Materials}

AIN-76A semipurified diet was obtained from Purina Test Diet (Richmond, IN). Grape preparation was provided by California Table Grape Commission (Fresno, CA).
Thiazolyl blue tetrazolium bromide (MTT) and trans-3,5,4'-trihydroxystilbene (resveratrol) were purchased from Sigma Chemical Company (St Louis, MO). Dulbecco's modified eagle's medium (DMEM), fetal bovine serum (FBS), trypsin EDTA and phosphate-buffered saline (PBS) were from Mediatech, Inc. (Herndon, VA). Dimethyl sulfoxide (DMSO) was obtained from Fisher Scientific (Fair Lawn, NJ). Cell proliferation enzyme linked immunosorbent assay (ELISA) kit was obtained from Roche Diagnostics $\mathrm{GmbH}$ (Mannheim, Germany).

\section{Freeze-dried grape extract preparation}

The preparation is composed of fresh grapes that have been frozen and ground with food-quality dry ice, freeze-dried and reground using good manufacturing practice for food products. As with fresh grapes, the preparation is known to contain resveratrol, flavans (including catechin), flavonols (including quercetin), anthocyanins, and simple phenolics as shown in Table 1.

\section{Animals}

Male Fischer rats (six-week-old) were purchased from Charles River (Wilmington, MA). Rats were housed in the College of Pharmacy animal room facility (temperature $22{ }^{\circ} \mathrm{C} \pm 1{ }^{\circ} \mathrm{C}$, humidity $40 \%$ to $60 \%$, and light from 6:00 AM. to 6:00 PM.) and provided food and water ad libitium.

\section{Colon carcinogenesis protocol}

The carcinogenesis protocol described by Bommareddy and colleagues ${ }^{20}$ was used to induce colon cancer. Six-weekold male Fischer rats were divided into two groups with 30 rats in each group as follows: Group 1: Control (AIN-76A diet); Group 2: Grape extract (AIN-76A containing 5\% w/w grape extract).

Table I Phytochemical analyzed in freeze-dried grape extract preparation (provided by California Table Grape Commission)

\begin{tabular}{lll}
\hline Compounds & Total & Individual \\
\hline Flavans & $17 \mathrm{mmol} / \mathrm{kg}$ & \\
Anthocyanins & $1.2 \mathrm{mmol} / \mathrm{kg}$ & \\
Flavonols & $80 \mu \mathrm{mol} / \mathrm{kg}$ & $68 \mu \mathrm{mol} / \mathrm{kg}$ \\
$\quad$ Quercetin & & $8 \mu \mathrm{mol} / \mathrm{kg}$ \\
Myricetin & & $3 \mu \mathrm{mol} / \mathrm{kg}$ \\
$\quad$ Kaempferol & \\
Stilbenes & & $38 \mu \mathrm{mol} / \mathrm{kg}$ \\
\hline
\end{tabular}

Notes: Flavans were analyzed by reaction with vanillin; anthocyanins were analyzed spectrophotometrically; flavonols and resveratrol were analyzed by high performance liquid chromatography after acid hydrolysis. 
Supplemented diet was prepared on a weekly basis by mixing the AIN-76A meal with an appropriate amount of grape extract in a mechanical mixer and was stored in the refrigerator.

After the rats from both groups had been on this diet for a week, they received subcutaneous injections of azoxymethane (15 mg/kg per week) for three weeks. The rats in all groups were weighted every week. The experiment was continued for 35 weeks after the last injection of azoxymethane. Then, the rats were anesthetized with ether. The intestinal tract was removed, flushed with $0.9 \% \mathrm{NaCl}$, and the number and sites of tumors recorded.

\section{Cell culture}

Human colonic adenocarcinoma (Caco-2) cell line was obtained from the American Type Culture Collection (ATCC; Manassas, VA). Cells were grown in DMEM, supplemented with $10 \%$ heat-inactivated FBS, 100 unit/mL of penicillin, and $100 \mu \mathrm{g} / \mathrm{mL}$ of streptomycin in a humidified atmosphere containing $5 \% \mathrm{CO}_{2}$ and $95 \%$ air at $37^{\circ} \mathrm{C}$.

\section{Resveratrol solution}

Resveratrol was dissolved in DMSO to make a concentration of $0.3 \mathrm{~mol} / \mathrm{L}$ as stock solution, and the stock solution was diluted in DMEM for producing different concentrations and immediately used. In all the assays, the final concentration of DMSO in DMEM was $0.33 \%$.

\section{Analysis of cell viability}

Cell viability was determined by MTT assay as described by Dariusz and colleagues. ${ }^{21}$ The cells were plated at a density of 5,000 cells/well in a 96-well plate. After 48 hours of cell attachment, cells were treated as either DMEM with $0.333 \%$ DMSO as control or various concentrations of resveratrol $(1 \mu \mathrm{M}-600 \mu \mathrm{M})$. After 72 hours of treatment, MTT stock solution $(5 \mathrm{mg} / \mathrm{mL})$ was diluted to 0.5 $\mathrm{mg} / \mathrm{mL}$ by DMEM medium and immediately used. The medium covering the cells was aspirated, and then cells were incubated with $50 \mu \mathrm{L}$ of $0.5 \mathrm{mg} / \mathrm{mL}$ MTT solution for four hours at $37^{\circ} \mathrm{C}$. Thereafter, $150 \mu \mathrm{L}$ of DMSO was added to each well and the plate was allowed to stand for one hour at $37^{\circ} \mathrm{C}$. Absorbance was measured by SpectraMax $\mathrm{M}_{2}$ microplate reader (Molecular Devices, Sunnyvale, CA) at $570 \mathrm{~nm}$, with the absorbance at $650 \mathrm{~nm}$ to correct for background in the presence of an appropriate blank (without cells). The blank reading was subtracted from experimental readings and cell viability was expressed as the percentage of the absorbance values of treated groups to untreated controls.

\section{Analysis of cell proliferation}

Cell proliferation was assessed by BrdU incorporation using colorimetric ELISA kit (Roche Diagnostics GmbH). Cells were plated and treated as described for the MTT assay. According to the protocol provided by the manufacturer, at the end of treatment, cells were first labeled with BrdU. Then, the labeling medium was removed. This was followed by adding FixDenat solution (Roche Molecular Biochemistry Mannheim, Germany) and incubating the plate for 30 minutes. Subsequently, FixDenat solution was removed and cells were incubated with anti-BrdU antibody peroxidase conjugate (anti-BrdU-POD) solution for about 90 minutes. Then, substrate solution was added to each well and incubated for 20 minutes. After addition of stop solution, the absorbance of samples was measured using a microplate reader (Molecular Devices) at $450 \mathrm{~nm}$ with the absorbance at $690 \mathrm{~nm}$ as reference. The blank (without cells) was performed in each experimental setup. The absorbance value of blank was subtracted from other experimental values and cell proliferation was expressed as the percentage of absorbance values of treated groups to untreated controls.

\section{Statistical analysis}

INSTAT software (GraphPad, San Diego, CA) was used to analyze the data. Chi-square was used for analyzing the data on tumor incidence. Student $t$-test was used to compare the tumor multiplicity and weight gain. ANOVA followed by Tukey post-hoc test was applied to compare the statistical difference of different resveratrol treatment groups with untreated controls for cell viability and cell proliferation studies. Significance in all the cases was considered at $\mathrm{p}<0.05$.

\section{Results \\ Weight gain}

Rats in both control and grape extract groups gained weight gradually over the 35-week experimental period. Average weight gain was not significantly different between control and grape extract groups (data not presented).

\section{Effects of grape extract on tumor incidence}

The incidence of tumor in the small intestine and colon is shown in Figure 2A. The incidence of tumors in the small 
intestine in control and grape extract groups was 35\% and $5.3 \%$, respectively. Dietary grape extract significantly $(\mathrm{p}<0.05)$ suppressed about $85 \%$ of small intestinal tumor incidence as compared to control group.

The incidence of colon tumors in control and grape preparation groups was $25 \%$ and $15.8 \%$, respectively; however, dietary grape extract did not significantly inhibit colon tumor incidence $(\mathrm{p}>0.0 .5)$ as compared to control group.

\section{Effects of grape extract on tumor multiplicity}

Tumor multiplicity of small intestine and colon is shown in Figure 2B. In the small intestine, control group had an average of 0.4 tumors per rat, whereas the grape extract group had an average of 0.05 tumors per rat. The mean number of tumors per rat in small intestine was significantly higher $(\mathrm{p}<0.05)$ in the control group than in the grape extract group.

The mean number of colon tumors per rats in the control and grape extract groups was 0.25 and 0.15 , respectively. Grape extract inhibited slightly the tumor multiplicity in colon compared with the control group. However, the inhibition was not statistically significant $(\mathrm{p}>0.05)$.

\section{Effects of resveratrol on cell viability in Caco-2 cells}

Since grape extract inhibits tumor incidence and tumor multiplicity in small intestine of rats, our aim for in vitro

A

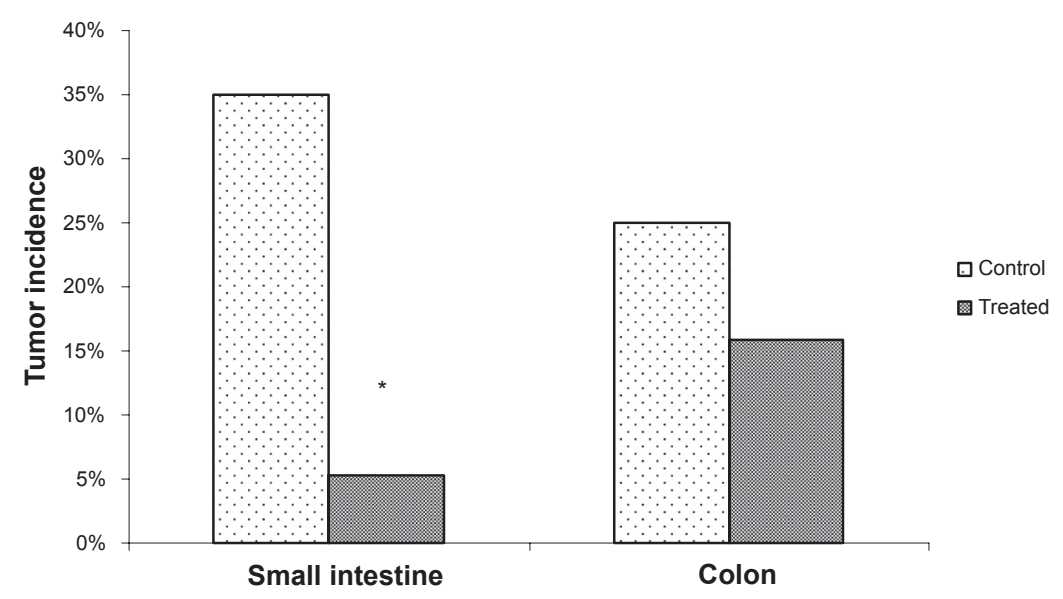

B

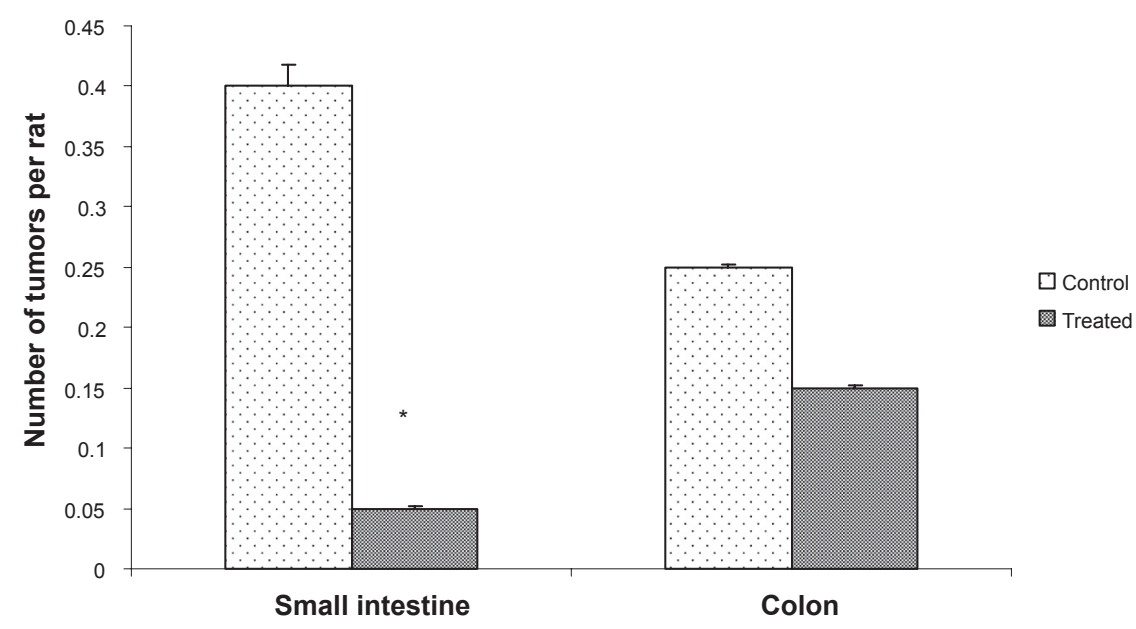

Figure 2 Effects of dietary grape extract on tumorigenesis in Fischer male rats. A. Effects of dietary grape extract on tumor incidence. Dietary grape extract significantly decreased the tumor incidence in small intestine. B. Effects of dietary grape extract on tumor multiplicity. Dietary grape extract significantly decreased the tumor multiplicity in small intestine. Notes: Data represents mean number of tumors per rats \pm standard deviation derived from 30 rats. *Means significant difference $(p<0.05)$ as compared to control. 
studies was to investigate whether resveratrol, a major component of grapes, could inhibit the growth of intestinal cancer cells, thus elucidating the mechanism of action of grape extract for in vivo studies. Therefore, effects of resveratrol on cell viability in Caco-2 cell line were assessed by utilizing MTT assay. Cells were treated with various concentrations of resveratrol $(1 \mu \mathrm{M}-600 \mu \mathrm{M})$ for 72 hours. As shown in Figure 3A, resveratrol treatment $(25 \mu \mathrm{M}-600 \mu \mathrm{M})$ significantly decreased $(\mathrm{p}<0.05)$ cell viability. Resveratrol treatment between $200 \mu \mathrm{M}$ and $400 \mu \mathrm{M}$ inhibited about $50 \%$ of cell viability in Caco-2 cells.

\section{Effects of resveratrol on cell proliferation in Caco-2 cells}

In similar treatments as above, the effects of resveratrol on cell proliferation in Caco- 2 cells were investigated by BrdU incorporation assay. Resveratrol treatment $(25 \mu \mathrm{M}-400 \mu \mathrm{M})$ significantly decreased $(\mathrm{p}<0.05)$ cell proliferation in Caco-2 cells as shown in Figure 3B. Moreover, resveratrol treatment

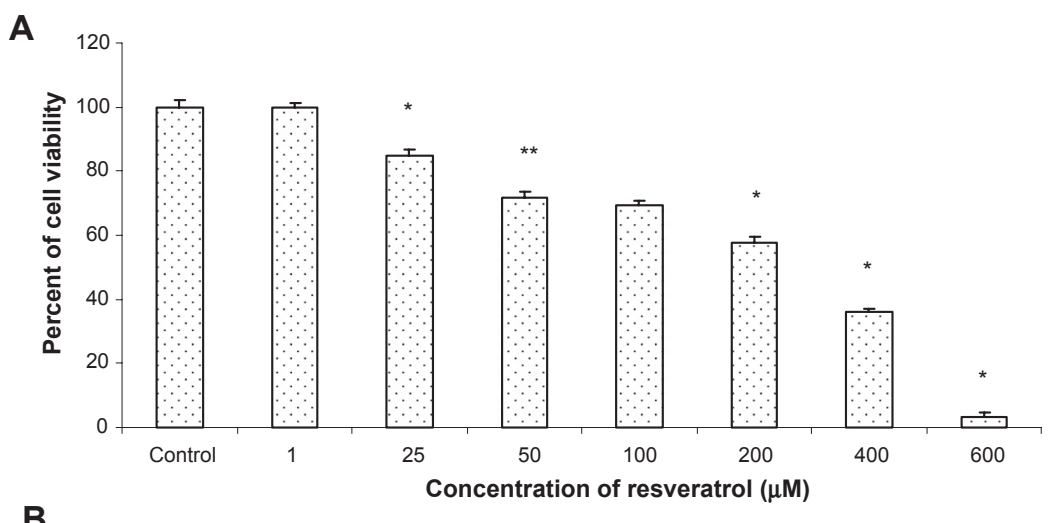

B

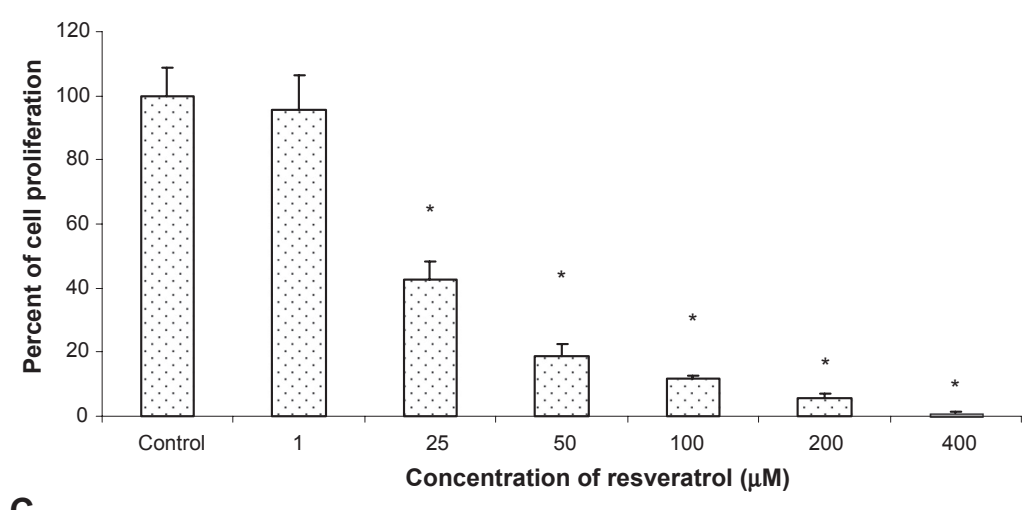

C

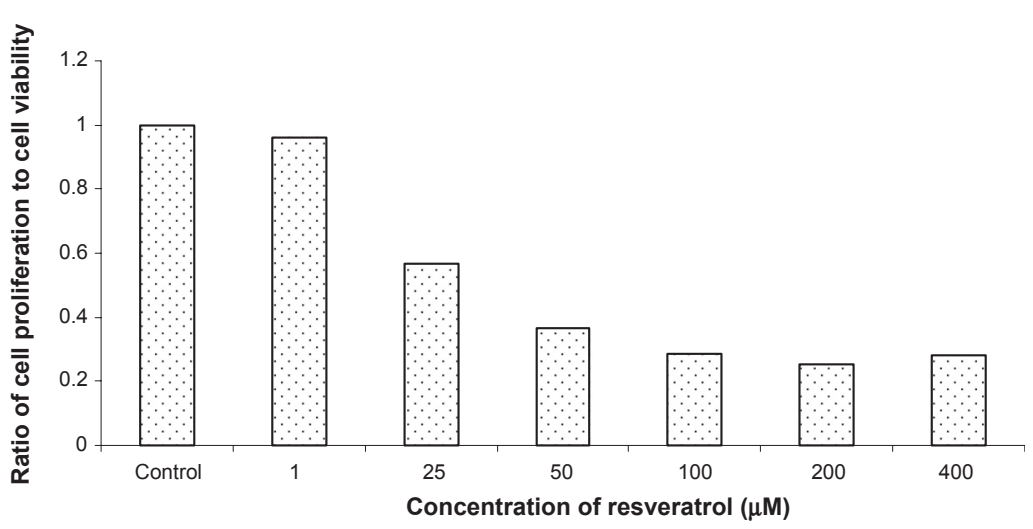

Figure 3 Effects of resveratrol on cell viability and cell proliferation in human colon adenocarcinoma (Caco-2) cells. Cells were treated with resveratrol ( $0 \mu \mathrm{M}-600 \mu \mathrm{M})$ for 72 hours. At the end of respective treatment, A. MTT and B. BrdU incorporation assays were performed as details in the materials and methods.Values of $\mathbf{A}$. MTT assay are mean \pm SE of eight replicates in each treatment, whereas values of $\mathbf{B}$. BrdU incorporation assay are mean \pm SE of four samples in each treatment. $\mathbf{C}$. Ratio of proliferating cells to viable cells.

Notes: *Indicates statistical significance $(p<0.05)$ in resveratrol-treated groups as compared to the control. 
in a concentration of $25 \mu \mathrm{M}$ inhibited more than $50 \%$ of cell proliferation compared with control. Overall results suggest that resveratrol treatment $(25 \mu \mathrm{M}-400 \mu \mathrm{M})$ for 72 hours could lead to a concentration-dependent inhibition of cell proliferation in Caco-2 cells.

Furthermore, the ratio of proliferative cells to viable cells for each treatment was calculated to investigate the role of either inhibition of cell viability or inhibition of cell proliferation caused by resveratrol on the decrease of cell growth in Caco- 2 cells. As shown in Figure 3C, the ratio of proliferative cells to viable cells for all the concentrations of resveratrol (1 $\mu \mathrm{M}-400 \mu \mathrm{M})$ was less than 1 . Thus, decreasing cell growth of Caco- 2 cells by resveratrol treatment was mainly due to the inhibition of cell proliferation rather than cell viability.

\section{Discussion}

Resveratrol has been reported to exhibit a wide range of biological and pharmacological properties. It has been speculated that resveratrol may act as an antioxidant and a cardioprotective agent by promoting nitric oxide production, inhibiting platelet aggregation, and increasing high-density lipoproteins..$^{3,22}$ Recently, chemoprevention of cancer by naturally occurring agents is gaining great attention by scientists and consumers as a plausible approach for the prevention of various cancers. Studies demonstrated that resveratrol can function as a chemopreventive agent for a wide variety of cancers including breast cancer, ${ }^{13,23}$ skin cancer, ${ }^{24}$ prostate cancer, ${ }^{25}$ and colon cancer. ${ }^{26}$ Animal studies on colon cancer also reported that dietary supplementation of resveratrol suppresses colonic tumor incidence in 1,2-dimethylhydrazine-treated rats $^{26}$ and resveratrol depresses azoxymethane-induced colon cancer development in male F344 rats for 100 days. ${ }^{27}$

In the present study, the effects of dietary grape extract ( $5 \%$; about $7 \mu \mathrm{g}$ resveratrol consumed daily), a dietary source of resveratrol on azoxymethane-induced colon intestinal cancer development in male Fischer rats were investigated. To our knowledge, this is the first study to show that dietary grape suppresses azoxymethane-induced colon cancer development in Fischer rats for 35 weeks. Azoxymethane-induced tumors share many histopathologic similarities with human tumors, and they often carry mutations of several genes such as $\beta$-catenins genes and $K$-ras. ${ }^{28}$ Our results revealed that dietary grape extract (about $7 \mu \mathrm{g}$ resveratrol consumed daily) resulted in an $85 \%$ and $88 \%$ inhibition of tumor incidence and tumor multiplicity in small intestine. Grape extract slightly decreased tumor incidence and tumor multiplicity in colon which did not reach statistical significance $(\mathrm{p}>0.05)$.
Cancer is a disease caused by uncontrolled cell proliferation. Accordingly, to understand the underlying mechanism(s) of the chemopreventive effects of dietary grape extract on rat colon cancer in vivo, the effects of resveratrol on cell viability and cell proliferation in Caco- 2 cell line was studied in vitro. Results showed that resveratrol treatment starting at $25 \mu \mathrm{M}$ significantly $(\mathrm{p}<0.05)$ decreased cell viability and cell proliferation of Caco- 2 cells. Moreover, the ratio of proliferative cells to viable cells for each resveratrol treatment suggested that inhibition of cell proliferation by resveratrol treatment is a main cause of decreasing the growth of colon tumor cells.

However, resveratrol resulted a significant decrease of cell proliferation in vitro at relatively high concentrations as compared to in vivo studies, and thus other mechanisms of chemoprevention of resveratrol may be involved. Recent reports demonstrated that resveratrol at lower concentrations inhibited Wnt signal throughput in colon-derived cells probably due to regulation of $\beta$-catenin localization. This activity may also contribute to the cancer prevention activity of resveratrol. ${ }^{29}$

Overall results suggested that dietary grape extract containing resveratrol could be chemopreventive for small intestinal cancer. The amount of resveratrol in red wine is $5 \mu \mathrm{mol} / \mathrm{L}$ to $13 \mu \mathrm{mol} / \mathrm{L} .{ }^{29}$ The high metabolic activity of rats, and extrapolation of animal data suggest that even one glass (100 mL, $\sim 1 \mu \mathrm{mol}, 250 \mu \mathrm{g}$ ) of red wine per day may provide protection against small intestinal cancer development in human. Furthermore, other components present in the dietary grape extract such as quercetin may also contribute to chemopreventive activity against intestinal cancer. ${ }^{30}$ Future investigations, including the effects of combination of resveratrol with other components such as quercetin present in the grape are needed to fully elucidate the effects of reseveratrol in red wine on intestinal cancer prevention.

\section{Acknowledgments}

The authors gratefully acknowledge California Table Grape Commission, Fresno, CA for providing freeze-dried grape extract for animal experiments.

\section{Disclosures}

The authors report no conflicts of interest in this work.

\section{References}

1. Creasy LL, Coffee M. Phytoalexin production potential of grape berries. J Am Soc of Hortic Sci. 1998;113:230-234.

2. Kopp P. Resveratrol, a phytoestrogen found in red wine. A possible explanation for the conundrum of 'French paradox'? Eur J Endocrinol. 1998;138:619-620. 
3. Das DK, Sato M, Ray PS, et al. Cardioprotection of red wine: role of polyphenolic antioxidants. Drugs Exp Clin Res. 1999;25:115-120.

4. Sato M, Ray PS, Maulik G, et al. Myocardical protection with red wine extract. J Cardiovasc Pharmacol. 2000;35:263-268.

5. Jang M, Cai L, Udeani GO, et al. Cancer chemopreventive activity of resveratrol, a natural product derived from grapes. Science. 1992;275:218-220.

6. Mohan J, Gandhi AA, Bhavya BC, et al. Caspase-2 triggers Bax-Bakdependent and -independent cell death in colon cancer cells treated with resveratrol. J Biol Chem. 2006;281:17599-17611.

7. van Ginkel PR, Sareen D, Subramanian L, et al. Resveratrol inhibits tumor growth of human neuroblastoma and mediates apoptosis by directly targeting mitochondria. Clin Cancer Res. 2007;13:5162-5169.

8. Park J, Woo KJ, Lee J, et al. Resveratrol induces pro-apoptotic endoplasmic reticulum stress in human colon cancer cells. Oncol Rep. 2007;18:1269-1273.

9. Marel A, Lizard G, Izard J, Latruffe N, Delmas D. Inhibitory effects of trans-resveratrol analogs molecules on the proliferation and the cell cycle proliferation of human colon tumoral cells. Mol Nutr Food Res. 2008;52:538-548.

10. Khanduja KL, Bharadwaj A. Kaushik G. Resveratrol inhibits $\mathrm{N}$-nitrosodiethylamine-induced ornathine decarboxylase and cyclooxygenase in mice. J Nutr Sci Vitaminol. 2004;50:61-65.

11. Wolter F, Ulrich S, Stein J. Molecular mechanism of the chemopreventive effects of resveratrol and its analogs in colorectal cancer: key role of polyamines? J Nutr. 2004;134:3219-3222.

12. Kundu JK, Shin YK, Kim SH, Surh YJ. Resveratrol inhibits phorbol ester-induced expression of COX-2 and activation of NF-kappaB in mouse skin by blocking IkappaB kinase activity. Carcinogenesis. 2006;27:1465-1474.

13. Tang H, Shih A, Cao HJ, Davis FB, Davis P, Lin H. Resveratrol-induced cyclooxygenase-2 facilitates p53-dependent apoptosis in human breast cancer cells. Mol Cancer Ther. 2006;5:2034-2042.

14. Eberhart CE, Coffey RJ, Radhika A, Giardiello FM, Ferrenbach S, DuBois RN. Up-regulation of cycloxygenase 2 gene expression in human colorectal adenomas and adenocarcinomas. Gastroenterology. 1994;107(4):1183-1188.

15. Thun MJ, Henley SJ, Patrono C. Nonsteroidal anti-inflammatory drugs as anticancer agents: mechanistic, pharmacologic, and clinical issues. J Natl Cancer Inst. 2002;94:252-266.

16. Sheng H, Shao J, Kirkland SC, et al. Inhibition of human cancer cell growth by selective inhibition of cyclooxygenase-2. J Clin Invest. 1997;99:2254-2259.

17. American Cancer Society Publication. Cancer facts and figures. 2008. Accessed on Mar 1, 2009. Available from: http://www.cancer.org/.
18. Willet WC. Balancing life-style and genomics research for disease prevention. Science. 2002;96:695-698.

19. Buolamwini JK. Cell cycle molecular target in novel anticancer drug discovery. Curr Pharm Des. 2000;6:379-392.

20. Bommareddy A, Arasada BL, Mathees DP, Dwivedi C. Chemopreventive effects of dietary flaxseed on colon tumor development. Nutr Cancer. 2006;54:216-222.

21. Dariusz S, Sarah JS, Richard HC, et al. An improved MTT assay. J Immunol Methods. 1993;157:203-207.

22. Vidavalur R, Otani H, Singal PK, Maulik N. Significance of wine and resveratrol in cardiovascular disease: French paradox revisited. Exp Clin Cardiol. 2006;11:217-225.

23. Ebert B, Seidel A. Lampen A. Phytochemical induce breast cancer resistance protein in Caco-2 cells and enhance the transport of benze[a]pyrene-3-sulfate. Toxicol Sci. 2007;96:227-236.

24. Aziz MH, Afaq F, Ahmad N. Prevention of ultraviolet-B radiation damage by resveratrol in mouse skin is mediated via modulation in surviving. Photochem Photobiol. 2005;81:25-31.

25. Harper CE, Patel BB, Wang J, Arabshahi A, Eltoum IA, Lamartiniere CA. Resveratrol suppresses prostate cancer progression in transgenic mice. Carcinogenesis. 2007;28:1946-1953.

26. Sengottuvelan M, Viswanathan P, Nalini N. Chemopreventive effect of trans-resveratrol- a phytoalexin against colonic aberrant crypt foci and cell proliferation in 1,2-dimethylhydrazine induced colon carcinogenesis. Carcinogenesis. 2006;27:1038-1046.

27. Tessitore L, Davit A, Sarotto I, Caderni G. Resveratrol depresses the growth of colorectal aberrant crypt foci by affecting bax and $\mathrm{p} 21^{\mathrm{CI}}$ expression. Carcinogenesis. 2000;21:1619-1622.

28. Athar M, Back JH, Tang X, et al. Kim Resveratrol: A review of preclinical studies for human cancer prevention. Toxicol Appl Pharmacol. 2008;224:274-283.

29. Hope C, Kestutis P, Marina P, et al. Low concentration of resveratrol inhibit Wnt singal throughput in colon-derived cells: immplications for colon cancer prevention. Mol Nutr Food Res. 2008;52(Suppl 1): S52-S61.

30. Mutoh M, Takahashi M, Fukuda K, et al. Suppression of cyclooxygenase-2 promoter-dependent transcriptional activity in colon cancer cells by chemopreventive agents with a resorcin-type structure. Carcinogenesis. 2000;21:959-963.

Martin AR, Villegas I, Sanchez-Hidalgo M, Lastra CA. The effects of resveratrol, a phytoalexin derived from red wine on chronic inflammation induced in an experimentally induced colitis model. Br J Pharmacol. 2006;147:873-885. 
